\title{
Correction to: Bariatric Surgery Does Not Improve Semen Quality: Evidence from a Meta-analysis
}

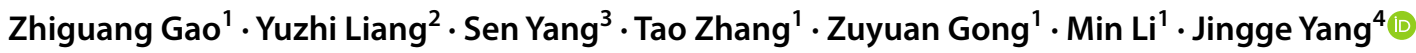 \\ Published online: 22 February 2022 \\ (c) The Author(s), under exclusive licence to Springer Science+Business Media, LLC, part of Springer Nature 2022
}

\section{Correction to: Obesity Surgery https://doi.org/10.1007/s11695-022-05901-8}

Some author affiliations were incorrectly assigned. Jingge Yang should have affiliation 4. Sen Yang should have affiliation 3 .

Publisher's Note Springer Nature remains neutral with regard to jurisdictional claims in published maps and institutional affiliations.

The original article can be found online at https://doi.org/10.1007/ s11695-022-05901-8.

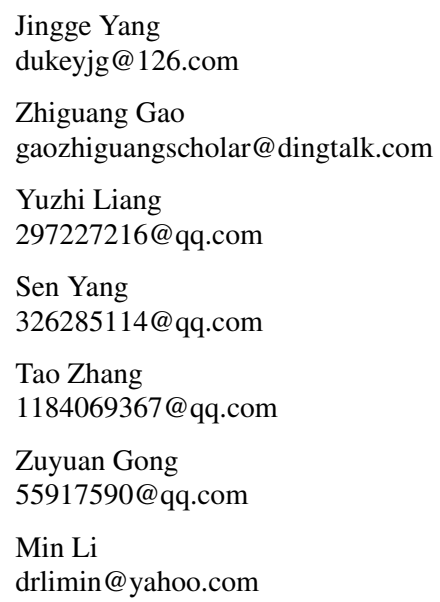

1 Department of Gastrointestinal Surgery, the affiliated Dongguan Shilong People's Hospital of Southern Medical University, Dongguan 523320, China

2 Department of Medical Imaging, the affiliated Dongguan Shilong People's Hospital of Southern Medical University, Dongguan 523320, China

3 Guangdong Medical University, Zhanjiang, Guangdong Province, China

4 Department of Gastrointestinal Surgery, First Affiliated Hospital of Jinan University, 613 Huangpu Avenue West, Guangzhou 510630, Guangdong Province, China 